\title{
Herramientas de diagnóstico para las intervenciones de regeneración urbana integrada en la ciudad consolidada: ejemplos de España
}

\author{
Eduardo de Santiago Rodríguez \\ Subdirección General de Políticas Urbanas. Ministerio de Fomento, España \\ Recibido: 6 de junio del 2017 / Aprobado: 13 de septiembre del 2017 \\ doi: 10.26439/limaq2018.n004.2252
}

El objetivo de este artículo es presentar el Observatorio de laVulnerabilidad Urbana en España como un ejemplo de herramienta para la evaluación y el diagnóstico de la vulnerabilidad urbana, previa a la regeneración de barrios en la ciudad consolidada a través de programas integrados que cuentan con financiación pública. El Observatorio de la Vulnerabilidad Urbana es un proyecto a largo plazo del Ministerio de Fomento del Gobierno de España en el que se pretende dar cabida a distintos estudios relacionados con la vulnerabilidad urbana en este país.

vulnerabilidad urbana, regeneración de barrios, evaluación, políticas públicas, segregación urbana, ciudad consolidada

Diagnostic tools for interventions of integrated urban regeneration in the consolidated city: examples from Spain

This article aims to present the Observatory of Urban Vulnerability in Spain as an example tool for the diagnosis and evaluation of urban vulnerability prior to the regeneration of neighborhoods in the consolidated city through integrated programs with public funding. The Observatory of Urban Vulnerability is a long-term project at the Ministry of Development in Spain, which intends to accommodate various studies related to the urban vulnerability in Spain.

urban vulnerability, neighborhood regeneration, evaluation, public policies, urban segregation, consolidated city 


\section{INTRODUCCIÓN: LA IMPORTANCIA DE LAS POLÍTICAS DE REHABILITACIÓN Y REGENERACIÓN URBANAS EN LOS CONTEXTOS ESPAÑOL Y EUROPEO}

En España, el artículo 47 de la Constitución establece el derecho a una vivienda digna y adecuada para todos los ciudadanos, derecho que se ha venido articulando mediante distintas políticas públicas, entre las cuales destacan las ayudas fiscales para la adquisición de viviendas y las ayudas directas o subvenciones dirigidas a facilitar la compra de viviendas de nueva construcción sometidas a algún régimen de protección pública. Estas ayudas públicas han sido reguladas mediante sucesivos planes estatales de vivienda de carácter cuatrienal que se han aprobado desde 1981 hasta el vigente plan 2013-2016. Sin perjuicio de algunas singularidades menores, todos estos planes mantuvieron a lo largo del tiempo un carácter unitario y constante tanto en su diseño como en su contenido. Por su parte, las 17 comunidades autónomas, en su propio ámbito competencial regional, fueron estableciendo ayudas adicionales, que completaban las estatales. Entre los elementos comunes que mantuvieron todos estos planes a lo largo del tiempo se pueden destacar el fomento de la producción de un volumen creciente de viviendas basándose en la ocupación de nuevos suelos y el crecimiento de las ciudades; y la apuesta, sobre todo, por la propiedad como forma esencial de acceso a la vivienda. Hasta el plan vigente, la rehabilitación tanto de viviendas aisladas como de viviendas en operaciones integradas a escala de barrio estaba presente en todos los planes, pero de forma muy minoritaria, mientras que se concentraban los recursos en las subvenciones para la construcción de viviendas nuevas.

La crisis económico-financiera internacional desatada en el 2007 afectó a España con especial virulencia y se manifestó con particular gravedad en el sector de la vivienda, en el cual se había generado una burbuja inmobiliaria o situación de fuerte desequilibrio, que llevó a la construcción de un número anual de viviendas muy por encima de las necesidades reales de la población. En efecto, tras casi una década (1997-2007) produciendo un número de viviendas desproporcionado, que casi alcanzó las 800000 anuales —equivalentes a la suma de las construidas anualmente en Francia y Alemania juntas- y que tuvo como efecto un endeudamiento excesivo de las familias (embarcadas en los préstamos hipotecarios suscritos para la compra de estas viviendas), se generó en España un significativo stock acumulado de viviendas acabadas, 
nuevas y sin vender, en torno a las 680000 unidades. Esto en fuerte contraste con las dificultades de los ciudadanos, especialmente de los sectores más vulnerables, quienes debido al incremento del desempleo, la precariedad y debilidad del mercado de trabajo, así como a la restricción de la financiación proveniente de las entidades bancarias encargadas de la concesión de nuevos préstamos hipotecarios, no pudieron acceder a una vivienda en propiedad o incluso sostener los pagos de los créditos hipotecarios previamente suscritos. Situación que provocó el desahucio o expulsión del propietario por no poder afrontar los pagos de las hipotecas pendientes.

En paralelo a ello, se constata la debilidad del mercado del alquiler de vivienda en España, sobre todo si se compara con el de otros países europeos. Las viviendas en alquiler suponen en España aproximadamente el $17 \%$ del total, frente al $83 \%$ de viviendas principales en propiedad. En Europa, en porcentajes medios, el mercado de la vivienda principal en alquiler representa el $38 \%$, frente al $62 \%$ de viviendas en propiedad.

Esta realidad económica, financiera y social imperante en España desde la crisis del 2007 aconsejaba un cambio de modelo que equilibrase ambas formas de acceso a la vivienda (propiedad y alquiler). Asimismo, que buscase la armonía entre la apuesta exclusiva por la construcción de nuevas viviendas, que se hizo durante los años de la burbuja (1997-2007), y el insuficiente mantenimiento y conservación del parque inmobiliario ya construido. Esto no solo porque la ciudad constituye un pilar fundamental para garantizar la calidad de vida de los ciudadanos y el disfrute por parte de ellos de un medio urbano adecuado, sino porque el sector de la rehabilitación, además, puede ofrecer un amplio marco para la reactivación del sector de la construcción, la generación de empleo y el ahorro, así como la eficiencia energética, en consonancia con las exigencias derivadas de las directivas europeas en la materia. Este importante papel de la rehabilitación y la regeneración urbanas integradas ya había sido puesto de manifiesto en el ámbito europeo en el 2010, en particular en la Declaración de Toledo (Unión Europea [UE], 2010) suscrita por los ministros responsables de desarrollo urbano de la UE. En ella destacaron la importancia de la regeneración urbana integrada y su potencial estratégico para un desarrollo urbano más inteligente, sostenible y socialmente inclusivo en Europa. 
En este contexto, el Gobierno español presentó en abril del 2013 un Plan Integral deVivienda y Suelo, con el fin de buscar un mayor equilibrio entre la vivienda en propiedad y la que está en alquiler, y de impulsar la rehabilitación, regeneración y renovación urbanas. El plan posteriormente se materializó a través de dos leyes - la Ley 4/2013, de Medidas de Flexibilización y Fomento del Alquiler; y la Ley 8/2013, de Rehabilitación, Regeneración y Renovación Urbanas - y un nuevo plan como instrumento de canalización de las ayudas públicas estatales, denominado "Plan estatal de fomento del alquiler de viviendas, la rehabilitación edificatoria, y la regeneración y renovación urbanas, 2013-2016", aprobado en el 2013 y prorrogado hasta el 2018. En consonancia con el contexto descrito anteriormente, los objetivos principales de este plan son los siguientes:

- Adaptar el sistema de ayudas a las necesidades sociales actuales y a la escasez de recursos disponibles al concentrarlo en dos ejes (fomento del alquiler y fomento de la rehabilitación, regeneración y renovación urbanas).

- Reforzar la cooperación y la coordinación interadministrativas, así como la corresponsabilidad en la financiación y en la gestión.

- Mejorar la calidad de la edificación y en particular contribuir a su buen estado de conservación, mejorar su eficiencia energética y promover su accesibilidad para todas las personas.

- Contribuir a la reactivación del sector inmobiliario desde los dos elementos motores señalados: el fomento del alquiler y el apoyo a la rehabilitación de edificios y a la regeneración urbana.

De los programas del plan relacionados con la rehabilitación destaca el Programa de Fomento de la Regeneración y Renovación Urbanas, destinado a la financiación de obras de rehabilitación en edificios y viviendas, así como de urbanización o reurbanización de espacios públicos $-\mathrm{y}$ en su caso de edificación en sustitución de edificios demolidos (renovación) - dentro de ámbitos de actuación previamente delimitados por las administraciones municipales o regionales. Su finalidad es, por tanto, la de mejorar los tejidos residenciales y recuperar funcionalmente conjuntos históricos, centros 
urbanos, barrios degradados y núcleos rurales, en especial aquellos que estén en peores condiciones o que presenten una mayor vulnerabilidad urbana.

\section{METODOLOGÍAS DE EVALUACIÓN Y DIAGNÓSTICO DE LA VULNERABILIDAD URBANA DESDE UNA PERSPECTIVA MULTIDIMENSIONAL}

Según el Diccionario de la Real Academia de la Lengua Española, el adjetivo vulnerable (del lat. vulnerabilis) significa 'que puede ser herido o recibir lesión, fisica o moralmente'; y según el Diccionario de uso del español, de María Moliner, significa que es 'susceptible de ser herido o vulnerado, en cualquier acepción, de recibir un daño o perjuicio, o de ser afectado, conmovido, convencido o vencido por algo'.

De modo que en su sentido etimológico, el término vulnerable expresa la susceptibilidad o posibilidad de ser herido, de recibir un daño o de ser afectado por alguna circunstancia. En un sentido urbano, y aplicado sobre un espacio social, la vulnerabilidad urbana se referiría a la potencialidad de que la población de un determinado espacio urbano concreto sea afectada por alguna circunstancia adversa. De este modo, el concepto aludiría no tanto a la existencia de una situación crítica constatada en la actualidad, sino más bien a unas determinadas condiciones de riesgo, fragilidad, desfavorecimiento o desventaja que harían posible la entrada en esa situación crítica. Así pues, este concepto estaría íntimamente relacionado con un punto de vista operativo, es decir, con la aplicación de actuaciones o medidas preventivas ${ }^{1}$ para que las potencialidades negativas no se materialicen.

Partiendo de la definición del concepto de la Organización de las Naciones Unidas (2003), Hernández Aja define la vulnerabilidad urbana de la siguiente manera:

Aquel proceso de malestar en las ciudades producido por la combinación de múltiples dimensiones de desventaja, en el que toda esperanza de movilidad social ascendente, de superación de su condición social de exclusión o

1 Frente a los que podrían ser otros puntos de vista de tipo curativo o paliativo, es decir, de actuación a posteriori. 
próxima a ella, es contemplada como extremadamente dificil de alcanzar. Por el contrario, conlleva una percepción de inseguridad y miedo a la posibilidad de una movilidad social descendente, de empeoramiento de sus actuales condiciones de vida. $(2007$, p. 8$)$

A partir de estas perspectivas, y con este planteamiento teórico, la elección de la metodología concreta para identificar de modo objetivo la vulnerabilidad de un espacio social urbano debe proponer respuestas a varias preguntas:

La primera, ¿cuál es el ámbito espacial de referencia (vecindario, barrio, distrito, ciudad, etcétera) donde se pretende analizar la vulnerabilidad? Esta cuestión resulta clave en la práctica, pues la respuesta queda condicionada por la disponibilidad o no de información estadística desagregada y asociada a la unidad de referencia elegida.

La segunda pregunta clave para definir una metodología de trabajo sería ¿cuáles son las variables significativas o indicadores elegidos para caracterizar la vulnerabilidad de modo objetivo? O dicho de otro modo, ¿cómo se mide la vulnerabilidad? En general, parece indiscutible que el enfoque de la vulnerabilidad debe realizarse desde la lógica de la complejidad ${ }^{2}$. Es decir, partir de una perspectiva multidimensional que, más allá de una sola variable simple (como puedan ser los ingresos familiares, el nivel de renta o la pobreza ${ }^{3}$ ), permita aproximarse de forma indirecta y a través de diferentes variables a la compleja problemática de las condiciones de fragilidad, desfavorecimiento o desventaja en los espacios sociales.

Desde este mismo punto de vista mutidimensional, el estudio pionero realizado por la OCDE en 1998 sobre distressed urban areas o 'áreas urbanas desfavorecidas' entendía que "distressed urban areas are portions of cities or

2 Una síntesis sobre la aplicación del pensamiento complejo a las ciencias sociales puede verse en Julio Alguacil Gómez (2000).

3 Aunque existe alguna definición consensuada internacionalmente sobre pobreza, como la de Eurostat - que define la tasa de personas que viven en situación de riesgo de pobreza como aquellas con "una renta disponible por debajo del umbral de riesgo de pobreza, que se establece en el $60 \%$ de la renta disponible del promedio nacional (incluidas las ayudas sociales)"-, existen infinidad de enfoques. Véase, a modo de síntesis en P. Spicker, S. Álvarez Leguizamón, y D. Gordon (2009). 
their suburbs, usually at the scale of residential neighbourhoods, in which social, economic and environmental problems are concentrated" (p. 15) ${ }^{4}$.

Partiendo, pues, de una definición compleja -multidimensional o multifactorial - de la vulnerabilidad, se trataría en primer lugar de identificar esa serie de variables capaces de traducir a términos mensurables cada uno de los aspectos o dimensiones que componen las múltiples facetas de esa aproximación compleja. En la mayoría de los casos este planteamiento resulta poco viable, no tanto desde el punto de vista teórico como desde el práctico, ante la ausencia de información disponible o la falta de homogeneidad de esta. En efecto, la escasa disponibilidad de datos suele condicionar de tal modo el listado de posibles variables que lo habitual resulta ser proceder de manera inversa: seleccionar sobre el conjunto de variables con información disponible y homogénea aquellas que permiten aproximarse y perfilar mejor cada una de las dimensiones múltiples que forman parte del concepto de vulnerabilidad planteado.

En algunos casos se ha llegado a proponer incluso la recomposición de este mosaico fragmentario de variables en un índice sintético, que se suele presentar bajo la denominación de índice de vulnerabilidad, o alguna otra similar. El problema estriba en que dicha recomposición, dada la naturaleza heterogénea de las variables que la integran ${ }^{5}$, se suele abordar mediante la asignación de pesos proporcionales, factores de conversión o ponderación a cada una de las distintas variables, para así poder componerlas en una sola expresión matemática. Desde el punto de vista matemático, ello supone la simplificación de un problema vectorial o multifactorial, convirtiéndolo previamente en uno escalar. Esto no siempre es posible y en relación con el concepto de vulnerabilidad al que nos estamos refiriendo puede ser cuestionable.

Finalmente, una tercera cuestión metodológica por responder sería esta: ¿cómo se determinan los valores de referencia o umbrales críticos para las variables identificadas a partir de los cuáles se estima que determinado espacio social es vulnerable? En una posterior derivada, esta cuestión se relaciona

4 Traducción: "Las áreas urbanas desfavorecidas son aquellas partes de las ciudades 0 de sus suburbios, habitualmente en la escala de los barrios de vivienda, en las que se concentran los problemas sociales, económicos y ambientales".

5 Como expresa un sabio refrán popular castellano, "No es posible sumar peras con manzanas". 
también con la posibilidad de establecer un gradiente o clasificación de las situaciones de vulnerabilidad. De nuevo nos encontramos con un problema matemático complejo, en el que tanto la fijación de los umbrales como la agrupación en clases son cuestiones abiertas que pueden estar dentro de los debates metodológicos actuales sobre técnicas de clasificación multicriterio, clustering, lógica borrosa, etcétera ${ }^{6}$.

\section{EL OBSERVATORIO DE LA VULNERABILIDAD URBANA EN ESPAÑA: VISIÓN PANORÁMICA DE SUS INSTRUMENTOS Y DE LA METODOLOGÍA UTILIZADA}

El Observatorio de la Vulnerabilidad (Ministerio de Fomento, 2011) es un proyecto a largo plazo que pretende dar cabida a los distintos estudios relacionados con este tema en España, y comprende diversos instrumentos, así como herramientas. A continuación, se detallan la metodología y los contenidos del Atlas de la Vulnerabilidad Urbana y del Análisis Urbanístico de Barrios Vulnerables, que son los dos componentes principales de la página web del Observatorio.

\section{El Atlas de la Vulnerabilidad Urbana en España}

El Atlas de la Vulnerabilidad Urbana en España (Ministerio de Fomento, 2015b) es una aplicación de cartografia dinámica en línea que permite analizar las múltiples dimensiones de la vulnerabilidad urbana, en el ámbito de sección censal, en todos los municipios de España. Se ha realizado a partir de los datos de los censos de población y viviendas del 2001 y $2011^{7}$.

Para cada una de las casi 35000 secciones censales de los más de 8000 municipios españoles, el Atlas de la Vulnerabilidad en España ofrece la posibilidad de realizar 145 mapas temáticos diferentes, personalizables por el usuario, bien mediante la representación por coropletas (112 mapas), bien mediante la representación por símbolos (33 mapas).

6 Una revisión del estado del arte puede verse en Ehrgott y Gandibleux (2002). Una aproximación más concreta al problema, en Hernández Aja y Vázquez Espí (2010).

7 En España, la única fuente estadística que dispone de información suficientemente desagregada en el ámbito espacial y que cubra todo el país es el censo, que se realiza cada 10 años. 
Los mapas se organizan en cuatro dominios:

Indicadores de vulnerabilidad urbana. Según la síntesis metodológica del Atlas (Ministerio de Fomento, 2015c), en esta categoría se presentan 57 mapas temáticos, correspondientes a 24 indicadores de vulnerabilidad urbana y a 33 variables estadísticas complementarias. Los indicadores de vulnerabilidad son datos relativos, expresados mediante porcentajes, y se representan con mapas de coropletas. Las variables estadísticas ofrecen valores absolutos (población, viviendas, etcétera) y se representan mediante símbolos, que pueden superponerse a las coropletas. Los mapas temáticos se agrupan en cuatro grandes temas: vulnerabilidad sociodemográfica ${ }^{8}$, socioeconómica ${ }^{9}$, residencia ${ }^{10}$ y subjetiva ${ }^{11}$. De los 24 indicadores, se consideran indicadores básicos de vulnerabilidad urbana (IBVU) los tres siguientes: porcentaje de población en paro, porcentaje de población sin estudios (que son comunes para el 2001 y el 2011) y porcentaje de población en viviendas sin servicio o aseo (como indicador de las carencias en las viviendas en el 2001), este último sustituido por porcentaje de viviendas en edificios en estado de conservación ruinoso, malo o deficiente para la fecha de referencia del 2011. Como se verá más adelante, estos IBVU se emplearán en el análisis urbanístico de barrios vulnerables en España para la identificación de los barrios vulnerables cuando estos indicadores superen, en las secciones censales que los componen, unos determinados valores de referencia con respecto a los valores medios nacionales.

8 Estos indicadores son porcentaje de población de 75 años y más; porcentaje de hogares unipersonales de mayores de 64 años; porcentaje de hogares con un adulto y un menor 0 más; porcentaje de población extranjera; y porcentaje de población extranjera infantil.

9 Porcentaje de población en paro (IBVU); porcentaje de población juvenil en paro; porcentaje de ocupados eventuales; porcentaje de ocupados no cualificados; y porcentaje de población sin estudios (IBVU).

10 Porcentaje de viviendas con menos de $30 \mathrm{~m}^{2}$; superficie media por habitante $\left(\mathrm{m}^{2}\right)$; porcentaje de población en viviendas sin servicio o aseo (IBVU); porcentaje de viviendas en edificios en mal estado de conservación; y porcentaje viviendas en edificios anteriores a 1951.

11 Porcentaje de población con problemas de ruidos exteriores; porcentaje de población con problemas de contaminación en su entorno; porcentaje de población con problemas de malas comunicaciones; porcentaje de población con problema de escasez de zonas verdes; porcentaje de población con problemas de delincuencia en su entorno. 
Todos los indicadores se han calculado a partir de los datos del Censo de Población y Vivienda, realizado por el Instituto Nacional de Estadística (INE).

En la figura 1 se presenta un ejemplo de visualización de la herramienta, en la que se representan dos indicadores en la ciudad de Barcelona (2011): el porcentaje de población extranjera en secciones censales y el número de viviendas menores de $30 \mathrm{~m}^{2}$. Permite constatar cómo los inmigrantes se concentraban en las viviendas de menor tamaño, especialmente en determinados barrios del centro urbano.

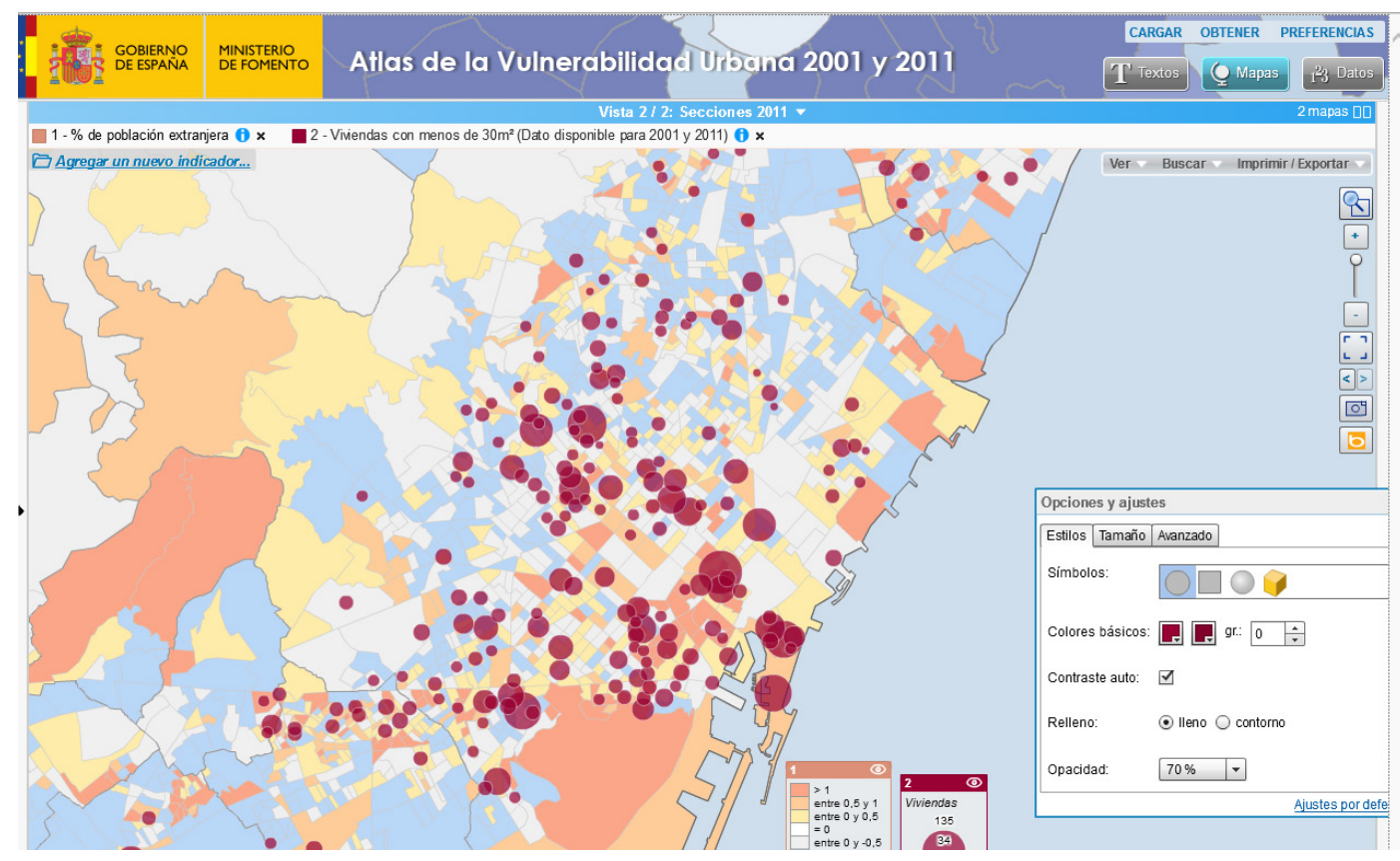

Figura 1

Ejemplo de visualización del Atlas de la Vulnerabilidad Urbana en España Fuente: http://atlasvulnerabilidadurbana.fomento.es 
Análisis contextual de la vulnerabilidad urbana. Este dominio permite realizar 72 mapas de coropletas, comparando los indicadores de vulnerabilidad de cada sección censal con el contexto municipal, regional (comunidad autónoma) o nacional; así se representa el número de veces en que el valor de un determinado indicador, de los 24 posibles, en una sección censal está por debajo o por encima del valor en el municipio, en la comunidad autónoma o en España.

En la figura 2 se presenta un ejemplo de análisis contextual, en el que se compara en dos fechas (2001 y 2011) el porcentaje de viviendas en mal estado de conservación con respecto a la media municipal en la ciudad de Madrid. De esta manera, es posible identificar los barrios donde ha habido una evolución positiva, para analizar posteriormente si coinciden o no con las áreas destinatarias de inversiones públicas en rehabilitación.

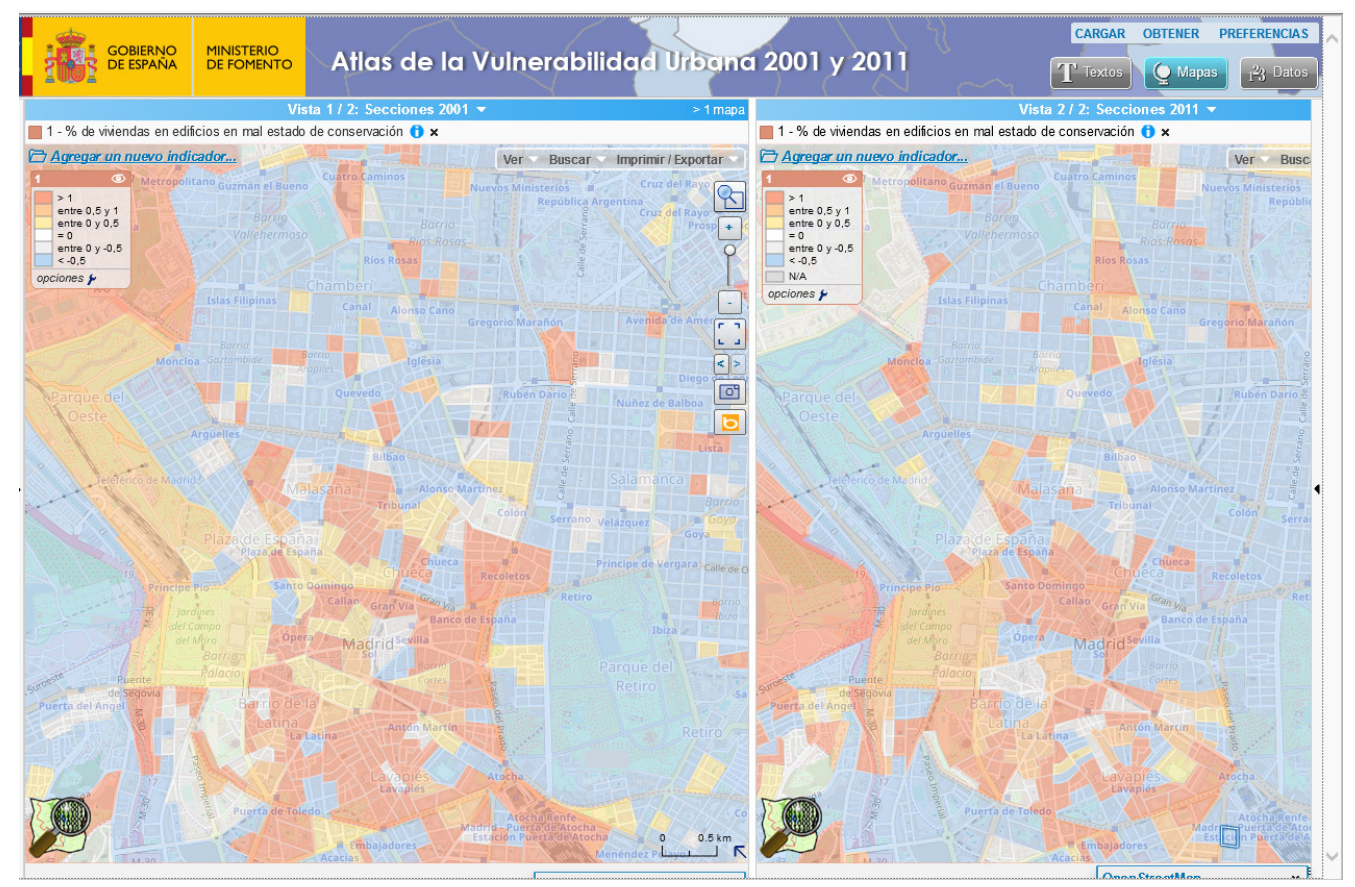

Figura 2

Ejemplo de análisis contextual diacrónico en España por medio del Atlas de la Vulnerabilidad Urbana

Fuente: http://atlasvulnerabilidadurbana.fomento.es 
Índices de desigualdad urbana. Este dominio ofrece para cada sección censal dos grandes índices (sintéticos) de desigualdad, calculados a partir de los tres IBVU:

- El índice de desigualdad socioeconómica (IDS), que se obtiene sumando los IBVU porcentaje de población en paro y porcentaje de población sin estudios, una vez que se han estandarizado por el método estadístico chi cuadrado y se ha aplicado posteriormente una transformación logarítmica para ajustar la distribución.

- El índice de desigualdad urbana (IDU), que se obtiene análogamente sumando los tres IBVU porcentaje de población en paro, porcentaje de población sin estudios y porcentaje de población en viviendas sin servicio o aseo, una vez que se han estandarizado por el método chi cuadrado y se ha aplicado posteriormente una transformación logarítmica para ajustar la distribución.

Cada uno de estos índices puede referenciarse con respecto al municipio, la comunidad autónoma o al conjunto de España.

Índices sintéticos de vulnerabilidad urbana (clasificación multicriterio). La clasificación multicriterio es una alternativa a la tradicional obtención de índices sintéticos a partir de la definición de funciones de tipo escalar que combinan los diferentes indicadores de vulnerabilidad mediante la asignación de un peso proporcional a cada uno de los indicadores ${ }^{12}$. Desde el punto de vista metodológico, la clasificación multicriterio consiste en agrupar en clases de equivalencia los elementos de un conjunto atendiendo al valor de $n$ índices o magnitudes y asignando el mismo valor global a cada uno de los elementos de cada clase ${ }^{13}$.

12 Como se ha dicho, el problema de este tipo de funciones es precisamente la subjetividad que existe en la asignación de los pesos proporcionales a cada indicador. La clasificación multicriterio permite eludir este problema.

13 La metodología detallada de la clasificación multicriterio puede consultarse en Ministerio de Fomento (2010). 
Aplicando una metodología de clasificación multicriterio, desarrollada específicamente para el Observatorio de la Vulnerabilidad, se obtienen cinco índices sintéticos de vulnerabilidad que componen los diferentes indicadores simples de vulnerabilidad manejados. Los cinco índices sintéticos finales corresponden a la clasificación multicriterio de las secciones atendiendo a subconjuntos parciales de criterios (agrupados en cuatro grandes categorías temáticas: clasificación según criterios sociodemográficos, según criterios socioeconómicos, según criterios residenciales y según criterios subjetivos) y a una clasificación multicriterio global, y englobando los 20 indicadores de vulnerabilidad considerados.

De este modo, los mapas incluidos en este dominio permiten representar la posición de cada sección censal española, determinada de acuerdo con cada una de estas cinco posibles clasificaciones multicriterio de la vulnerabilidad urbana. Teniendo en cuenta que cada clasificación se realiza sobre dos universos diferentes (al clasificar todas y cada una de las secciones censales con respecto al universo nacional o al universo de cada comunidad autónoma), se pueden obtener un total de 10 mapas temáticos.

En la figura 3 se presenta, a modo de ejemplo, la visualización correspondiente al índice sintético de vulnerabilidad urbana del centro de la ciudad de Madrid. Este se ha obtenido mediante la clasificación multicriterio, en la que se comparan los valores de cada sección con los valores medios del país. Esto permite identificar los barrios que están peor clasificados con respecto a la media nacional y apreciar una correspondencia casi exacta con los tejidos del casco histórico. 


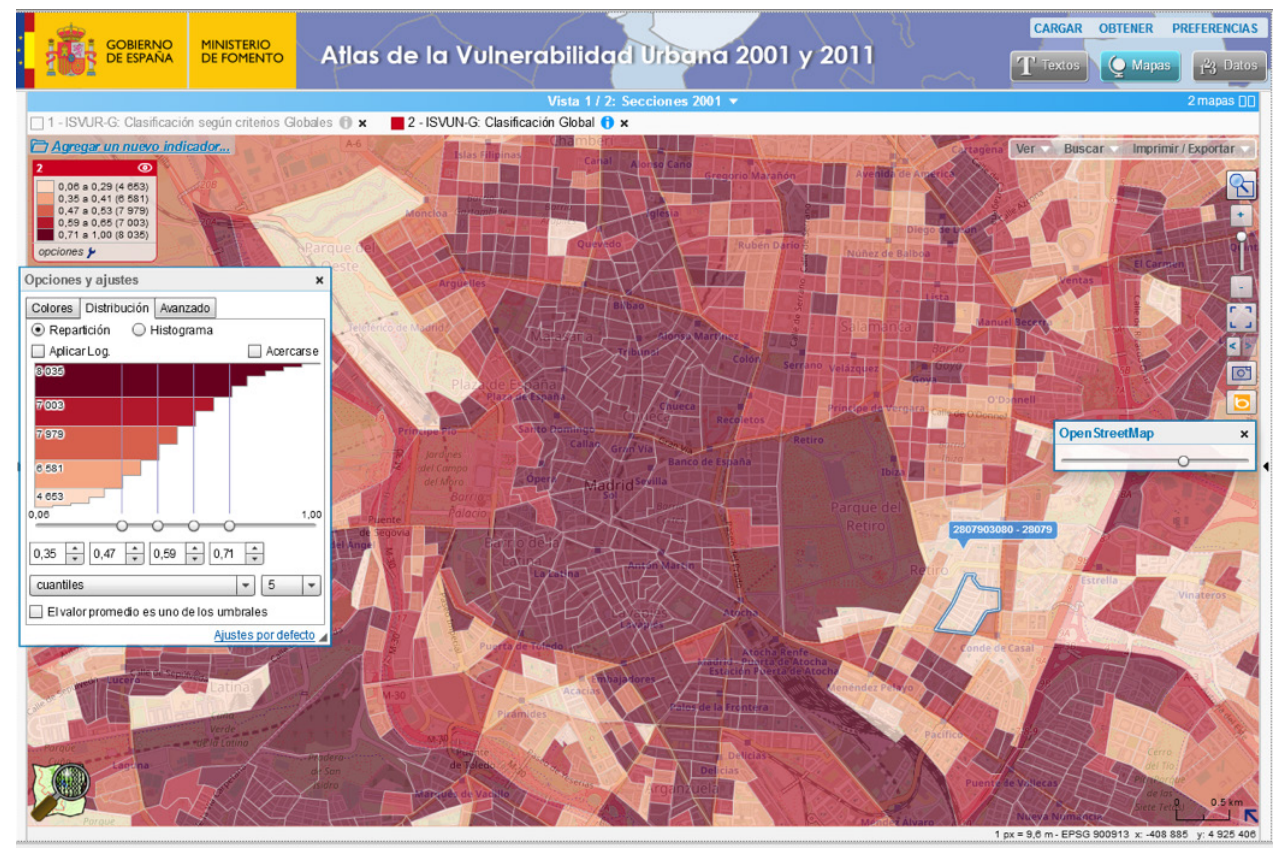

Figura 3

Atlas de la Vulnerabilidad Urbana en España: índice sintético de vulnerabilidad urbana en la ciudad de Madrid Fuente: http://atlasvulnerabilidadurbana.fomento.es

\section{El Atlas de la Edificación Residencial en España (200I y 20II)}

El Atlas de la Edificación Residencial (Ministerio de Fomento, 2015a) es una aplicación web de estructura y características similares al Atlas de la Vulnerabilidad, pero en este caso centrada exclusivamente en las particularidades de los edificios de vivienda. Ofrece 66 indicadores y 25 variables cualitativas agrupadas en cuatro dominios: características básicas de los edificios y las viviendas; características de uso, propiedad y régimen de tenencia; estado de conservación y disponibilidad de instalaciones; y caracterización urbanística. Estos indicadores permiten conocer detalladamente aspectos como superficie de las viviendas, estado de 
conservación, altura de los edificios, accesibilidad y existencia de ascensor, dotación de calefacción, número de ocupantes, etcétera.

El análisis urbanistico de barrios vulnerables en España (I99I, 200I, 2006 y 20II)

Según la metodología del trabajo (Ministerio de Fomento, 2015a) de la batería completa de indicadores disponibles en los Atlas de la Vulnerabilidad, a efectos del análisis urbanístico de barrios vulnerables en España, se consideran tres IBVU, que son los siguientes ${ }^{14}$ :

- Porcentaje de población en paro, común para los años 1991, 2001 y 2011

- Porcentaje de población sin estudios, común también para 1991, 2001 y 2011

- Un tercer IBVU, representativo de las carencias o problemas en las viviendas. Dado que la información disponible sobre este aspecto en los diferentes censos nacionales de 1991, 2001 y 2011 ha ido variando por cuestiones metodológicas de los propios censos, este indicador en cada uno de los años ha sido el siguiente:

- En 1991 se tomaron tres indicadores representativos de las carencias en las viviendas: porcentaje de viviendas sin agua corriente, porcentaje de viviendas sin retrete dentro de la vivienda y porcentaje de viviendas sin baño o ducha dentro de la vivienda.

- En el 2001 se tomó el porcentaje de población en viviendas sin retrete.

- En el 2011 se tomó el porcentaje de viviendas en edificios en estado de conservación ruinoso, malo o deficiente.

14 Se escogieron estos tres indicadores porque son los más similares a los requeridos por el estudio comparativo de la OCDE, realizado en 1998, cuya continuación a lo largo del tiempo ha dado lugar a la creación del observatorio. 
Además, se ha trabajado con un indicador complementario, introducido a partir del 2006, el de inmigración. Este pretende evaluar las características contextuales de dicha población extranjera, sin entender en ningún caso que dicha condición implique necesariamente la vulnerabilidad de dicha población.

La vulnerabilidad se detecta cuando alguno de los IBVU en una sección censal supera unos determinados valores de referencia con respecto al valor medio nacional. Ello no impide que en un análisis más detallado, como el que puede realizar el usuario de forma dinámica y personalizada con los Atlas de Vulnerabilidad, puedan detectarse otros ámbitos con vulnerabilidad contextual, resultante de comparar los indicadores con los valores de referencia regional o municipal.

Como criterio general, el umbral de vulnerabilidad se establece al superar 1,5 veces el valor correspondiente a nivel nacional, pero en su determinación se consideran también otras cuestiones. De este modo, finalmente la elección de este umbral o valor de referencia se ha realizado de forma cuidadosa en cada uno de los diferentes catálogos y según una metodología detallada que puede consultarse en los informes o síntesis metodológicas correspondientes a cada una de las fechas (1991, 2001, 2006 y 2011). En la tabla 1 se resumen los valores nacionales y de referencia para los tres IBVU en el Análisis Urbanístico de Barrios Vulnerables en España, referido a los censos de 1991, 2001 y 2011. También se señalan con color los umbrales usados en cada fecha para la identificación de los barrios vulnerables (criterio de vulnerabilidad).

Una vez identificadas las secciones censales que, en cada una de las fechas de estudio, superan los umbrales de referencia (para uno o varios de los tres IBVU), se procede a agrupar estas secciones en áreas estadísticas vulnerables $(\mathrm{AEV})$ y posteriormente en barrios vulnerables (BBVV).

En el caso del indicador de inmigración (disponible para el 2006 y el 2011) se opera de forma análoga. 


\begin{tabular}{|c|c|c|c|c|c|c|}
\hline \multirow{2}{*}{$\begin{array}{l}\text { Indicadores básicos de } \\
\text { vulnerabilidad urbana }\end{array}$} & \multicolumn{2}{|l|}{1991} & \multicolumn{2}{|l|}{2001} & \multicolumn{2}{|l|}{2011} \\
\hline & $\begin{array}{l}\text { Valor } \\
\text { España }\end{array}$ & $\begin{array}{l}\text { Criterio } \\
\text { vulnerabilidad }\end{array}$ & $\begin{array}{l}\text { Valor } \\
\text { España }\end{array}$ & $\begin{array}{l}\text { Criterio } \\
\text { vulnerabilidad }\end{array}$ & $\begin{array}{l}\text { Valor } \\
\text { España }\end{array}$ & $\begin{array}{l}\text { Criterio } \\
\text { vulnerabilidad }\end{array}$ \\
\hline IBVU paro & 19,30 & Mayor de 28 & 14,20 & $\begin{array}{l}\text { Mayor o igual } \\
\text { a } 21,30\end{array}$ & 29,64 & $\begin{array}{l}\text { Mayor o igual } \\
\text { a } 42,33\end{array}$ \\
\hline IBVU estudios & 18,78 & Mayor de 28 & 15,30 & $\begin{array}{l}\text { Mayor o igual } \\
\text { a } 22,95\end{array}$ & 10,92 & $\begin{array}{l}\text { Mayor o igual } \\
\text { a } 16,38\end{array}$ \\
\hline \multicolumn{7}{|l|}{ IBVU vivienda } \\
\hline $\begin{array}{l}\text { Porcentaje de } \\
\text { viviendas sin agua } \\
\text { corriente }\end{array}$ & 0,66 & Mayor de 2 & 0,59 & $x$ & 0,16 & $x$ \\
\hline $\begin{array}{l}\text { Porcentaje de } \\
\text { viviendas sin retrete } \\
\text { dentro de la vivienda }\end{array}$ & 2,84 & Mayor de 3 & $x$ & $x$ & 0,58 & $x$ \\
\hline $\begin{array}{l}\text { Porcentaje de } \\
\text { viviendas sin baño o } \\
\text { ducha dentro de la } \\
\text { vivienda }\end{array}$ & 4,51 & Mayor de 5 & $x$ & $x$ & 0,51 & $x$ \\
\hline $\begin{array}{l}\text { Porcentaje de } \\
\text { población en } \\
\text { viviendas sin servicio } \\
\text { o aseo }\end{array}$ & $x$ & $x$ & 1,00 & $\begin{array}{l}\text { Mayor o igual } \\
\text { a } 2,00\end{array}$ & $x$ & $x$ \\
\hline $\begin{array}{l}\text { Porcentaje de } \\
\text { viviendas en edificios } \\
\text { en estado ruinoso, } \\
\text { deficiente o malo }\end{array}$ & $10,39 \%$ & $x$ & 10,09 & $x$ & 6,99 & $\begin{array}{l}\text { Mayor o igual } \\
\text { a } 17,5\end{array}$ \\
\hline
\end{tabular}

Tabla 1. Cuadro sintético con los valores de los indicadores básicos de vulnerabilidad urbana y los criterios de corte seleccionados en cada fecha de referencia Fuente: Ministerio de Fomento (2016) 
A partir de estos indicadores, disponibles para todas las secciones censales del país, el proceso de delimitación y ajuste espacial preciso de los barrios vulnerables se desarrolla solo en las ciudades españolas de más de 50000 habitantes y en las capitales de provincia. A continuación, se describe una síntesis metodológica de los estudios referidos a 1991 y 2001 (Ministerio de Fomento, 2016), en el que el sistema de trabajo es el siguiente:

- Primero, se realiza un análisis del Censo de Población y Vivienda en la fecha correspondiente al año de estudio (1991 o 2001), a través de los valores de referencia que se toman como los tres indicadores básicos de vulnerabilidad urbana: porcentaje de población en paro, porcentaje de población analfabeta y sin estudios, e indicador de carencias en la vivienda (porcentaje de viviendas sin agua corriente, inodoro o retrete, baño o ducha en 1991; porcentaje de población sin servicio o aseo, en el 2001; porcentaje de viviendas en edificios en estado de conservación ruinoso, deficiente o malo, en el 2011), con el fin de localizar las secciones censales cuyos indicadores (uno o más) superan los umbrales de referencia. Estas pasan a ser estudiadas más detalladamente mediante su delimitación sobre el plano, donde conforman las áreas estadísticas vulnerables.

- En una segunda fase, y tras haber realizado una entrevista a los técnicos municipales de cada ciudad y el correspondiente trabajo de campo, se realiza un análisis de tipo urbanístico con el fin de definir la delimitación final precisa de cada barrio vulnerable.

- Por último, se envía a los técnicos municipales un borrador con la documentación elaborada en cada ciudad, para que completen y corrijan, si es necesario, la información referente a su municipio.

- La información correspondiente a cada barrio vulnerable se organiza mediante una ficha urbanística de descripción del barrio, que trata de reflejar su realidad actual tanto urbana como social, y una ficha estadística (referida al área estadística vulnerable asociada) con toda la información estadística y los indicadores de vulnerabilidad. Esta última se presenta a modo de ejemplo en la figura 4. 


\begin{tabular}{|c|c|c|c|}
\hline 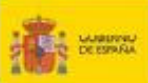 & Droums & $\begin{array}{l}2001 \\
\text { Lavapies }\end{array}$ & $\begin{array}{r}\text { Madrid } \\
28079007\end{array}$ \\
\hline
\end{tabular}

\section{Ficha estadística}

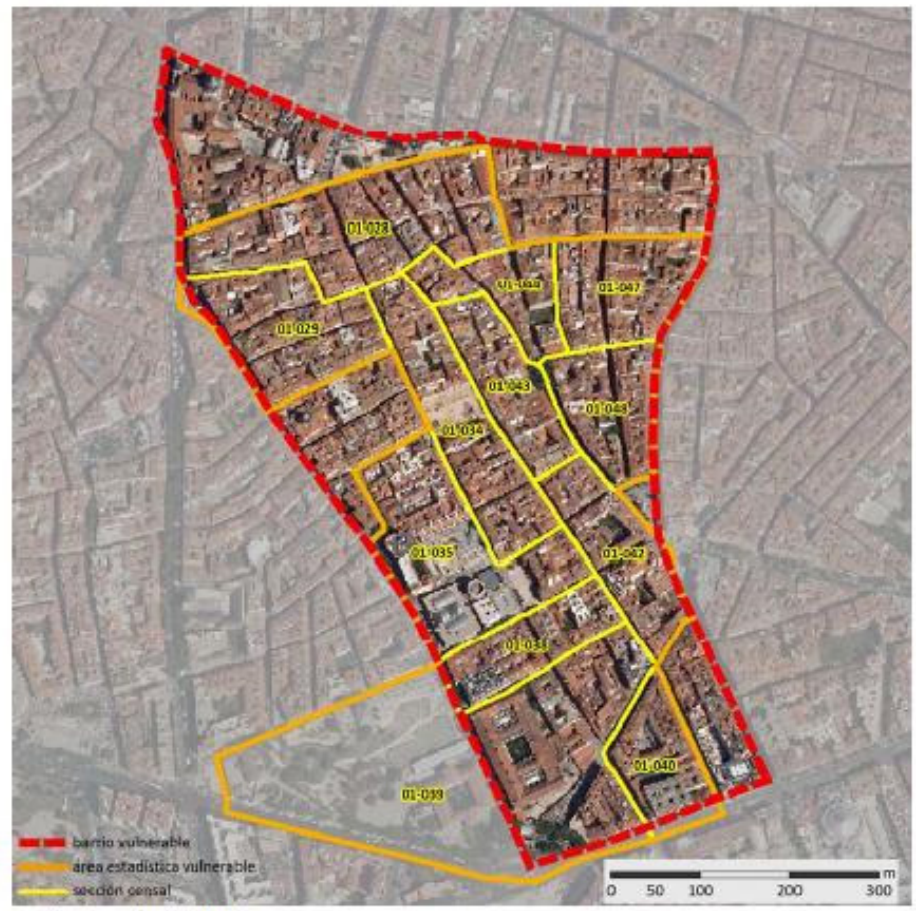

Localización en detalle del AEV

\section{Datos básicos}

Población del AEV

Viviendas

Densidad población (pob/ $/ \mathrm{Km}^{2}$ ) 44.835,35

Densidad vivienda (Viv/Ha)

Superficie (Ha)
13.343

9.135

307,11

29,75

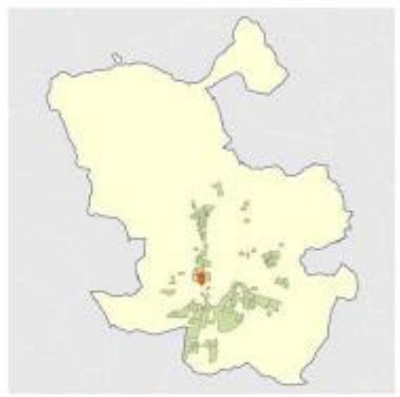

Localización del AEV dentro del término municipal

\section{Valores de referencia}

\begin{tabular}{|c|c|}
\hline Índice de estudios (a) & $15,19 \%$ \\
\hline Índice de viviendas (b) & $5,52 \%$ \\
\hline Índice de paro & $18,21 \%$ \\
\hline
\end{tabular}

(a) Porcentaje de población analfabeta y sin estudios

(b) Porcentaje de población residente en viviendas famillises sin sevicio o aseo dentro de la vivienda

(c) Tasa de paro

\begin{tabular}{|r|l|}
\hline $22,95 \%$ & valores superiones en un $50 \%$ a la media nacional \\
\hline $2,00 \%$ & valores dos veces superiores a la media nacional \\
\hline $21,30 \%$ & valores superiores en un $50 \%$ a la media nacional
\end{tabular}

(continúa) 
(continuación)

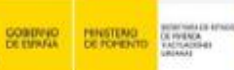

\begin{tabular}{lrr} 
DATOS SOCIODEMOGRÁFICOS & Valores absolutos \\
Población (2001) & & \\
\hline Población total & 13.343 & 10,45 \\
Población < 15 años & 1.395 & 69,34 \\
Población 15 - 64 años & 9.252 & 19,87 \\
Población > 64 años & 2.651 & 43,73 \\
Tasa de dependencia (1) & &
\end{tabular}

Hogares (2001)

$\begin{array}{lrl}\text { Hogares totales } & 5.979 & \\ \text { Hogares con menores de } 16 \text { años } & 670 & 11,21 \\ \text { Hogares con } 4 \text { miembros o más } & 1.006 & 16,83 \\ \text { Tamafio medio del hogar (2) } & 2,23 & \end{array}$

Nivel educativo (2001)

$\begin{array}{lll}\text { Población sin estudios (3) } & 1.808 & 15,19 \\ \text { Población con estudios primarios } & 2.464 & 20,70 \\ \text { Población con estudios secundarios } & 5.364 & 45,06 \\ \text { Población con estudios universitarios } & 2.267 & 19,05\end{array}$

Situación laboral (2001)

\begin{tabular}{|c|c|c|}
\hline Población en edad laboral (4) & 9.252 & 69,34 \\
\hline Tasa de actividad (5) & & 63,01 \\
\hline Tasa de ocupación (6) & & 51,53 \\
\hline
\end{tabular}

Edificación (2001)

Edificios totales

Edificios con más de 30 años (7)

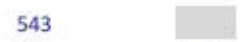

Edificios con más de 50 años (8)

87,48

Edificios con más de 80 años (9)

$\begin{array}{ll}475 & 87,48 \\ 445 & 81,95\end{array}$

$362 \quad 66,67$

Vivienda (2001)

$\begin{array}{lrrr}\text { Viviendas totales } & & 9.138 & \\ \text { Viviendas principales } & (10) & 5.979 & 65,43 \\ \text { Viviendas secundarias } \quad(11) & 940 & 10,29 \\ \text { Viviendas vacías (12) } & 2.064 & 22,59 \\ \text { Viviendas en propiedad } \quad(13) & 3.557 & 59,49 \\ \text { Viviendas en alquiler (14) } & 2.166 & 36,23 \\ \text { Viviendas en edif. de 4 plantas o más sin ascensor } & (15) & 4.032 & 70,67 \\ \text { Superficie media útil por vivienda }\left(\mathrm{m}^{2}\right) & 30,98 & \\ \text { Densidad de viviendas (viv/Ha) } & 307,11 & \end{array}$

Figura 4

Ejemplo de ficha estadística del Catálogo de Barrios Vulnerables en España en el 2001, correspondiente al barrio de Lavapiés (Madrid)

Fuente: http://www.fomento.gob.es/MFOM.BarriosVulnerables/ 


\section{CONCLUSIONES: LA CONTRIBUCIÓN POTENCIAL DEL OBSERVATORIO DE LA VULNERABILIDAD EN ESPAÑA A LOS PROGRAMAS DE REGENERACIÓN URBANA}

El Observatorio de la Vulnerabilidad Urbana es una plataforma de análisis y reflexión, que permite coordinar los esfuerzos de las distintas administraciones públicas españolas en la lucha contra la vulnerabilidad; además, tiene una clara vocación operativa al servicio de los programas de regeneración urbana integrada.

Esta vocación queda manifiesta en el Visor del Catálogo de Barrios Vulnerables, de acceso público en internet, que permite visualizar los barrios identificados como vulnerables sobre un mapa o sobre una fotografia aérea y descargar las fichas informativas (urbanística y estadística) referidas a cada uno de ellos. El cuadro de diálogo sirve para seleccionar y filtrar diferentes opciones de búsqueda: por barrios o por ciudades, por año o por nivel de vulnerabilidad. Existen dos series de indicadores de vulnerabilidad: los tres IBVU (paro, nivel de estudios y carencias en las viviendas), disponibles para 1991, 2001 y 2011; y la serie del indicador de inmigración, disponible para el 2006 y el 2011. Debajo del mapa se ofrece una tabla de resultados con la información de los elementos seleccionados y visualizados en el mapa, que puede también ser descargada para su consulta. La figura 5 muestra un ejemplo de visualización.

El catálogo también permite superponer las delimitaciones de los barrios vulnerables de 1991, 2001, 2006 y 2011, de modo que posibilita un análisis evolutivo de las dinámicas urbanas ${ }^{15}$.

El observatorio pretende que todas estas funcionalidades sirvan de ayuda a las distintas administraciones públicas españolas para la definición de los programas de mejoramiento barrial en barrios vulnerables. En particular, puede ser una herramienta muy apropiada de diagnóstico urbano, ya que permite analizar un conjunto muy amplio de indicadores y compararlos con

15 Desafortunadamente, solo es posible un análisis gráfico en el que se comparen los planos de cada fecha para el indicador seleccionado, pues en España la delimitación de las secciones censales puede variar de un periodo intercensal a otro. Por tanto, no es posible hacer análisis estadísticos rigurosos agregados de la evolución de los indicadores en cada sección, solamente análisis gráficos. 


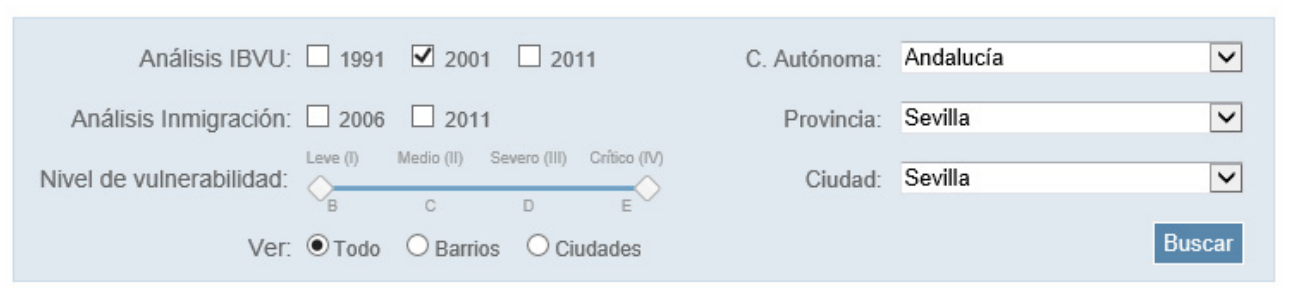

Ver mapa en pantalla completa

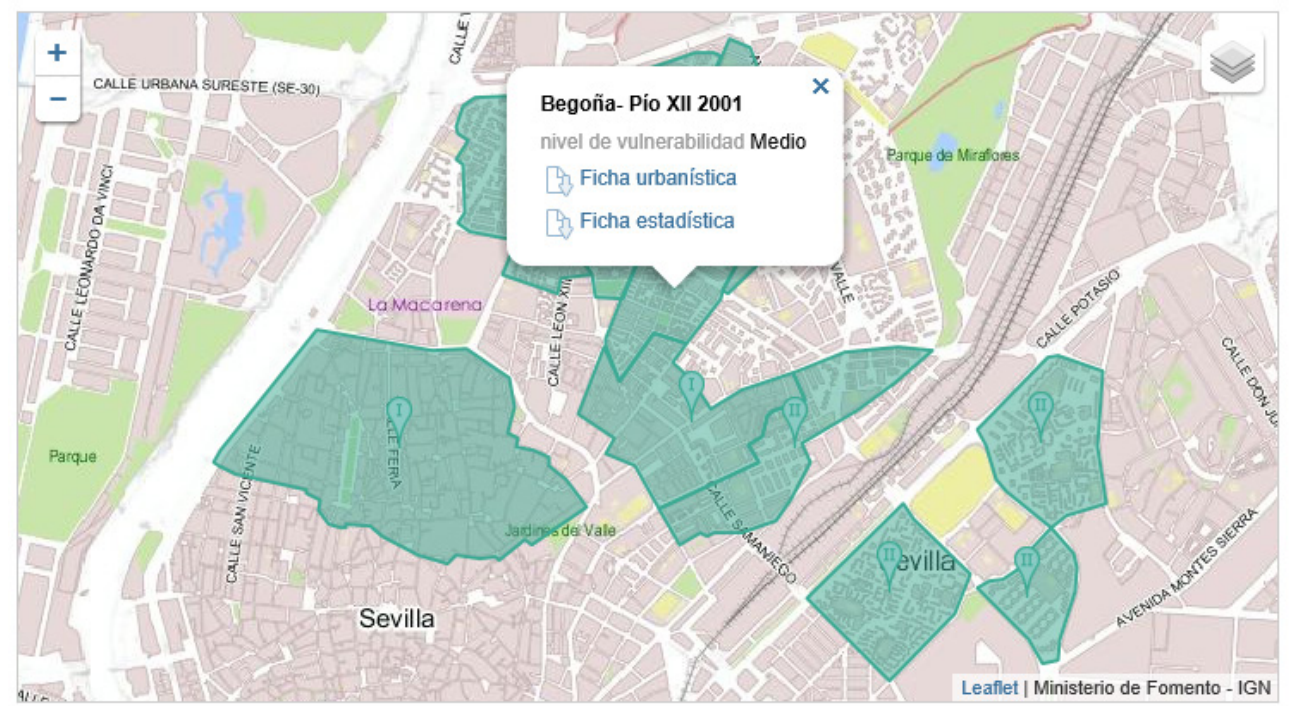

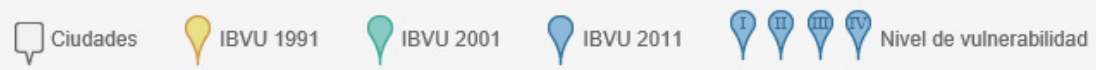

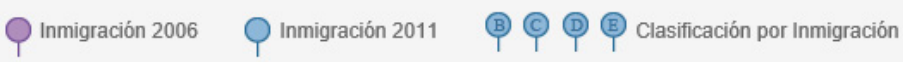

Figura 5

Visualización del Catálogo de Barrios Vulnerables de la ciudad de Sevilla (centro urbano) en el 2001 Fuente: http://www.fomento.gob.es/MFOM.BarriosVulnerables/

las medias nacional, regional y municipal. El Programa de Fomento de la Regeneración y Renovación Urbanas del vigente plan estatal exige, además de la delimitación espacial precisa del ámbito de regeneración y renovación urbanas por la administración competente, presentar una memoria- 
programa, que debe contener un diagnóstico sobre la situación social, económica y ambiental del ámbito. Dicho diagnóstico estará justificado a partir de indicadores e índices estadísticos objetivos en relación con la media municipal, autonómica y estatal, para lo cual las herramientas presentadas pueden ser de gran ayuda.

El observatorio también permite comparar la situación de vulnerabilidad de los barrios a lo largo del tiempo con los programas de regeneración urbana financiados con los planes estatales. Es posible, por tanto, la evaluación del impacto de estos sobre los barrios de las ciudades españolas.

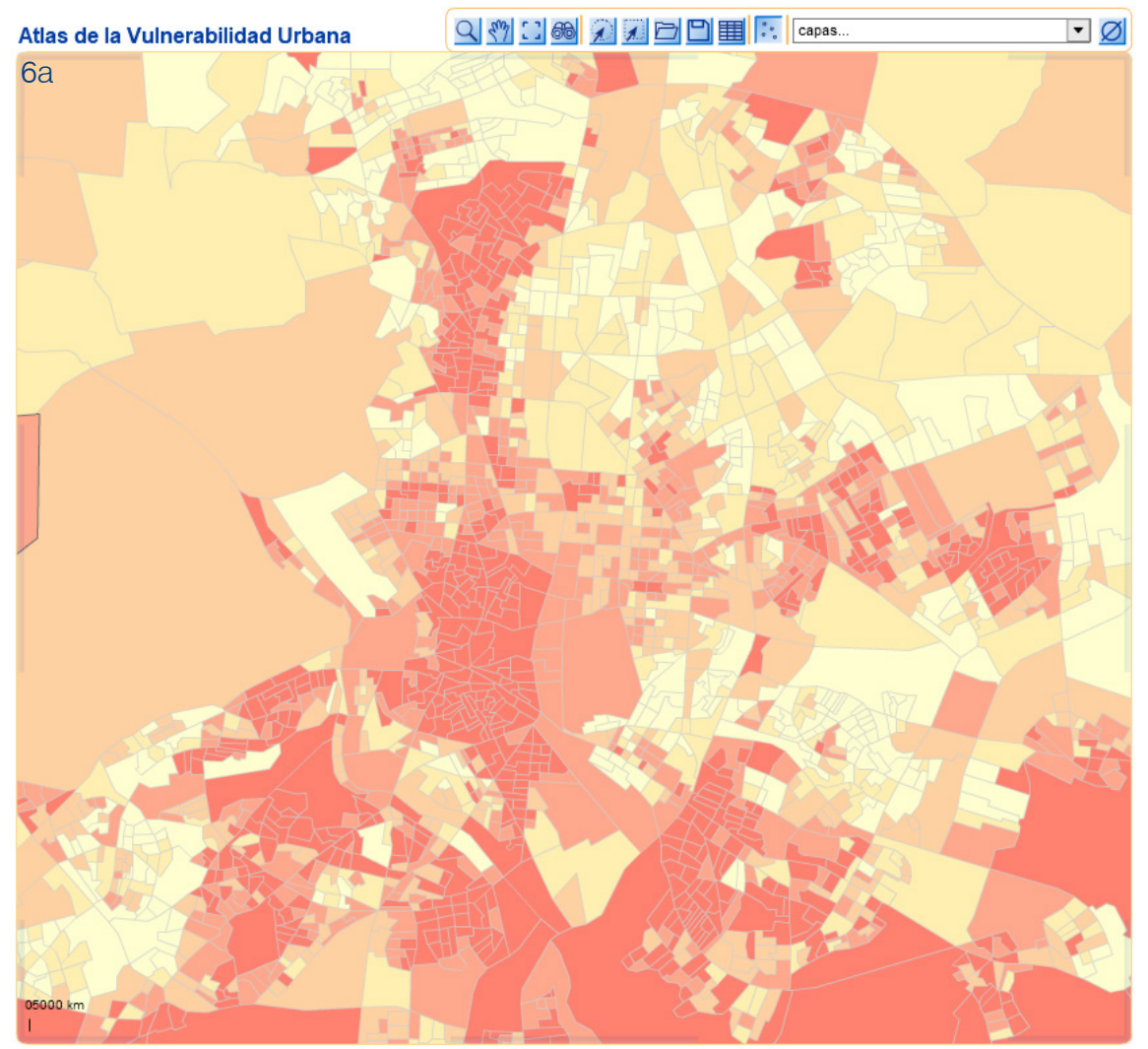

Figura 6

6a. Î́ndice sintético de vulnerabilidad urbana: clasificación multicriterio a nivel nacional 


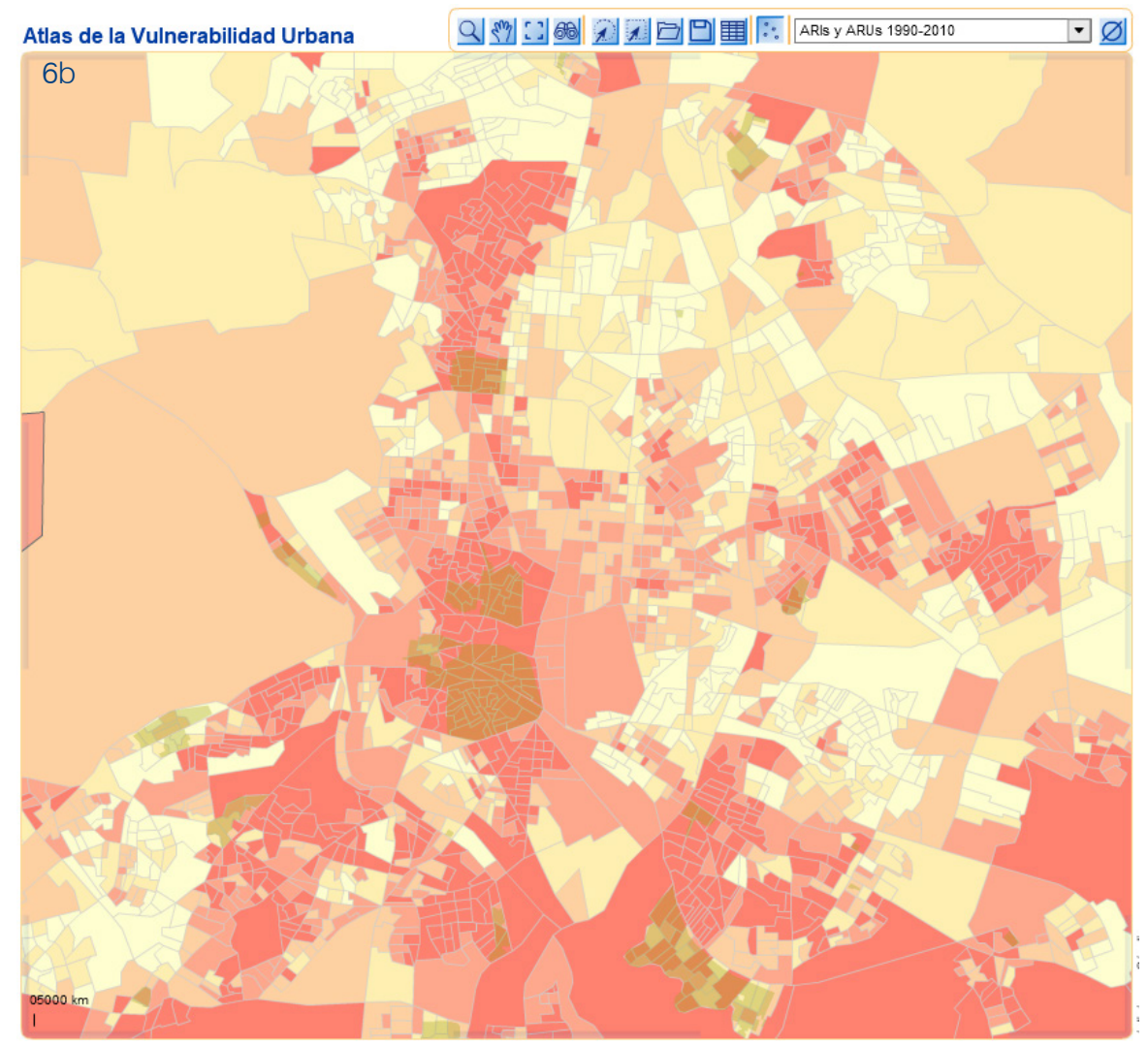

Figura 6

6b. Barrios vulnerables identificados a partir del censo de 2001 


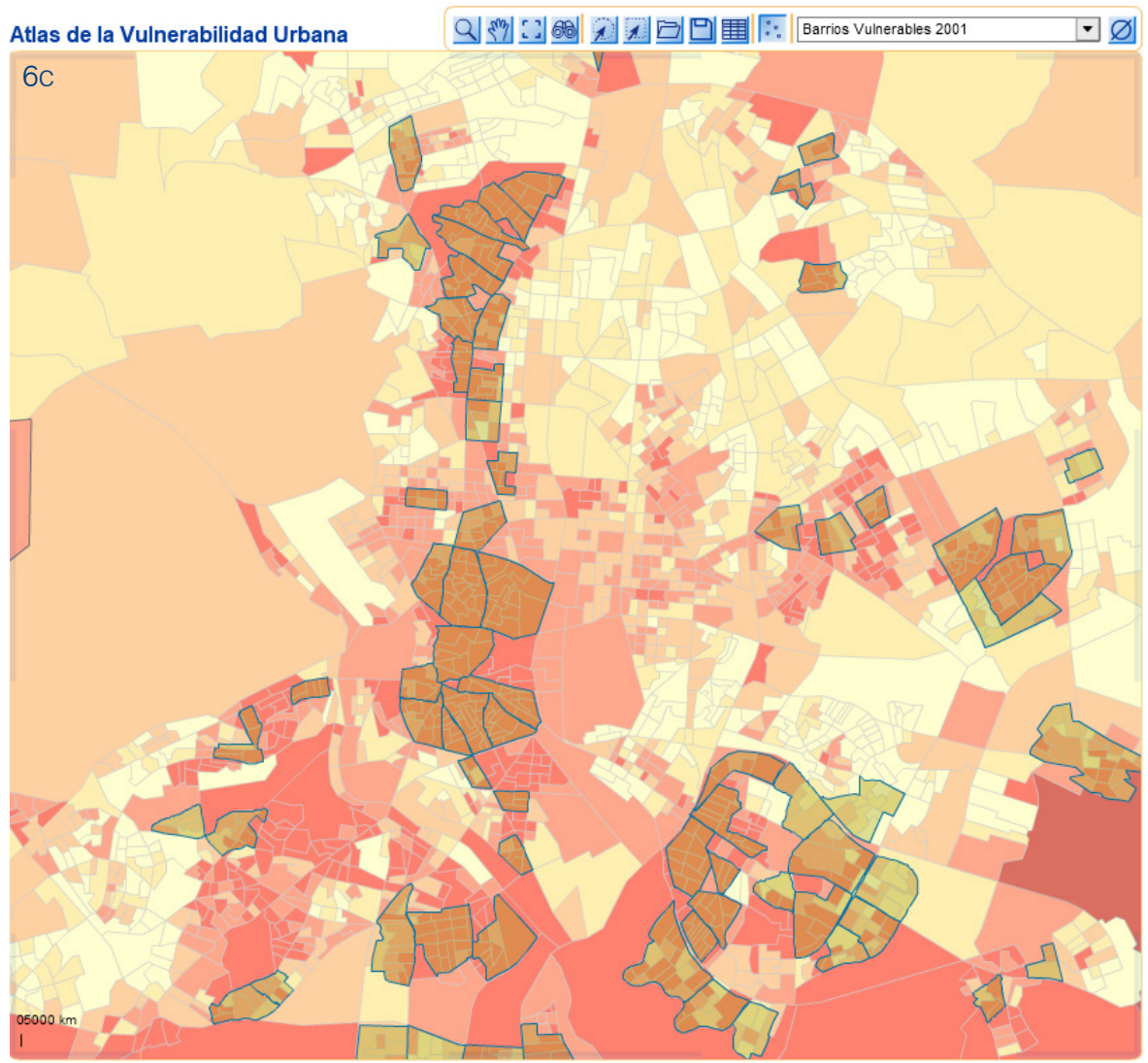

Figura 6

6c. Áreas de rehabilitación integral (ARI) y áreas de renovación urbana (ARU) financiadas por los planes estatales de vivienda 1990-2010 en la ciudad de Madrid Fuente: http://atlasvulnerabilidadurbana2001.fomento.es/ 
Dada la novedad de la herramienta, todavía no ha sido posible analizar su potencial de ayuda en la toma de decisiones en los ámbitos local o nacional. No obstante, como ejemplo de este potencial, en la figura 6 se ha presentado un análisis de este tipo, para la ciudad de Madrid, en el que se puede comprobar como sí existe una fiel correspondencia entre los barrios vulnerables identificados por el estudio y el índice sintético de vulnerabilidad urbana (elaborado con la clasificación multicriterio) a nivel nacional, el cual permite reconocer las secciones más vulnerables (el color más oscuro indica mayor vulnerabilidad). Además, se observa cómo la comparación de estos barrios con las áreas que han recibido financiación pública indica la concentración de los recursos públicos sobre los barrios vulnerables del centro de la ciudad y la atención en mucho menor medida de los barrios periféricos. De este modo, la herramienta permite constatar esta carencia y ofrece un respaldo técnico a la necesidad de corregir esta situación al financiar actuaciones en estos barrios de la periferia madrileña.

\section{REFERENCIAS}

Alguacil Gómez,J. (2000). Calidad de vida y praxis urbana: nuevas iniciativas de gestión ciudadana en la periferia social de Madrid. Colección Monografia: 179. Madrid: Centro de Investigaciones Sociológicas.

Arias Goytre, F. (Director) (2000) La desigualdad urbana en España. Madrid: Ministerio de Fomento (Centro de Publicaciones).

Ehrgott, M., y Gandibleux, X. (2002): Multiple Criteria Optimization: State of the Art Annotated Bibliographic Survey. En International Series in Operation Research and Management Science. Dordrecht: Kluwer Academic Publishers Group.

Eurostat. (s. f.) EU statistics on income and living conditions (EUSILC) methodology. Recuperado de http://ec.europa.eu/eurostat/ statistics-explained/index.php/EU_statistics_on_income_and_living_ conditions_(EU-SILC)_methodology

Hernández Aja, A. (2007). Áreas vulnerables en el centro de Madrid. Cuadernos de Investigación Urbanística, (53). 
Hernández Aja, A., y Vázquez Espí, M. (2010). Clasificación multicriterio: una regla simple para tratar problemas complejos. Biblioteca $C F+S$. Recuperado de http://habitat.aq.upm.es/gi/mve/2010-cm.pdf

Ministerio de Fomento. (2010). Anexo I. Conceptos clasificación multicriterio. En Análisis urbanístico de barrios vulnerables en España. Recuperado de https://www.fomento.gob.es/NR/rdonlyres/ F5A51594-86A6-4ED0-9A93-103EE1BA65DA/111347/ANEXOS_ COMPLETO.pdf

Ministerio de Fomento. (2011): Observatorio de la Vulnerabilidad Urbana. Recuperado de http://www.fomento.gob.es/MFOM/ LANG_CASTELLANO/DIRECCIONES_GENERALES/ARQ_ VIVIENDA/SUELO_Y_POLITICAS/OBSERVATORIO/

Ministerio de Fomento. (2015a). Atlas de la Edificación Residencial en España 2001 y 2011. Metodología, contenidos y créditos. Recuperado de https://www.fomento.gob.es/MFOM/LANG_CASTELLANO/ DIRECCIONES_GENERALES/ARQ_VIVIENDA/SUELO_Y_ POLITICAS/OBSERVATORIO/AtlEdiResEsp/

Ministerio de Fomento. (2015b): Atlas de la Vulnerabilidad Urbana en España. Recuperado de https://www.fomento.gob.es/MFOM/ LANG_CASTELLANO/DIRECCIONES_GENERALES/ARQ_ VIVIENDA/SUELO_Y_POLITICAS/OBSERVATORIO/Atlas_ Vulnerabilidad_Urbana/

Ministerio de Fomento. (2015c). Atlas de la Vulnerabilidad Urbana en España 2001 y 2011. Metodología, contenidos y créditos. Recuperado de https:// www.fomento.gob.es/NR/rdonlyres/40668D5E-26B6-4720-867F 286BD55E1C6B/135960/20160201METODOLOGIAATLASVULN ERABILIDAD2001Y2011.pdf

Ministerio de Fomento. (2016). Análisis urbanístico de barrios vulnerables en España. Síntesis metodológica general del catálogo de barrios vulnerables e indicadores básicos de vulnerabilidad urbana. Recuperado de https:// www.fomento.gob.es/MFOM.BarriosVulnerables/static/adjunto /Sintesis_metodologica_general_catalogo_bbvv.pdf 
Organización de las Naciones Unidas.Departamento de Asuntos Económicos y Sociales. (2003). Informe sobre la situación social del mundo 2003. Vulnerabilidad social: Fuentes y desafios. Nueva York: Organización de las Naciones Unidas.

OCDE. (1998). Integrating Distressed Urban Areas. París: OCDE Publishing. https://doi.org/10.1787/9789264162884-en

Spicker, P., Álvarez Leguizamón, S., y Gordon, D. (Eds.). (2009). Pobreza: Un glosario internacional. Buenos Aires: Consejo Latinoamericano de Ciencias Sociales.

Unión Europea. Ministros responsables de Desarrollo Urbano. (22 de junio del 2010). Declaración de Toledo. Recuperado de http://ec.europa.eu/ regional_policy/archive/newsroom/pdf/201006_toledo_declaration_ es.pdf 